\title{
Constructing Cartesian Splines
}

\author{
H.R.N. van Erp ${ }^{*}, 1$, R.O. Linger ${ }^{2}$ and P.H.A.J.M. van Gelder ${ }^{1}$ \\ ${ }^{I}$ TU Delft Structural Hydraulic and Probabilistic Design, TU Delft, Delft, The Netherlands \\ ${ }^{2}$ Medical Sciences, RuG, Groningen, The Netherlands
}

\begin{abstract}
We introduce here Cartesian splines or, for short, C-splines. C-splines are piecewise polynomials which are defined on adjacent Cartesian coordinate systems and are $C^{r}$ continuous throughout. The $C^{r}$ continuity is enforced by constraining the coefficients of the polynomial to lie in the null-space of some smoothness matrix $H$. The matrix-product of the null-space of the smoothness matrix $H$ and the original polynomial base results in a new base, the so-called Cspline base, which automatically enforces $C^{r}$ continuity throughout. In this article we give a derivation of this $\mathrm{C}$-spline base as well as an algorithm to construct $\mathrm{C}$-spline models.
\end{abstract}

Keywords: Splines, cartesian, regression analysis, explicit base.

\section{INTRODUCTION}

We introduce here Cartesian splines or, for short, Csplines. C-splines are piecewise polynomials which are defined on adjacent Cartesian coordinate systems and are $C^{r}$ continuous throughout. The $C^{r}$ continuity is enforced by constraining the coefficients of the polynomial to lie in the null-space of some smoothness matrix $H$. The matrixproduct of the null-space of the smoothness matrix $H$ and the original polynomial base results in a new base, the socalled C-spline base, which automatically enforces $C^{r}$ continuity throughout. The idea of using the null-space of some smoothness matrix $H$ has been taken from the Bspline literature, where piecewise polynomials are defined on adjacent triangular Barycentric coordinate systems [1-3]. It turns out that $\mathrm{C}$-spline bases have a particular simple form. This makes it possible to give an explicit formulation of general C-spline bases. In this article we will give a general outline how to enforce continuity constraints by way of the smoothness matrix $H$. We then show how these constraints lead us to the C-spline base. Then we will give the explicit algorithm for constructing a bivariate $\mathrm{C}$-spline base and show how to use this base to construct a C-spline model.

\section{PIECEWISE POLYNOMIALS}

We start with the bivariate Cartesian $x, y$-coordinate system. We partition this initial coordinate system with origin $O=(0,0)$ in two adjacent coordinate systems, each with its own origin, $O=(0,0)$ and $\tilde{O}=(0,0)$. The geometry in terms of $x$ and $y$ may be depicted as:

*Address correspondence to this author at the Structural Hydraulic and Probabilistic Design, TU Delft, Delft, The Netherlands; Tel: 06-30883099; E-mail: H.R.N.vanErp@tudelft.nl

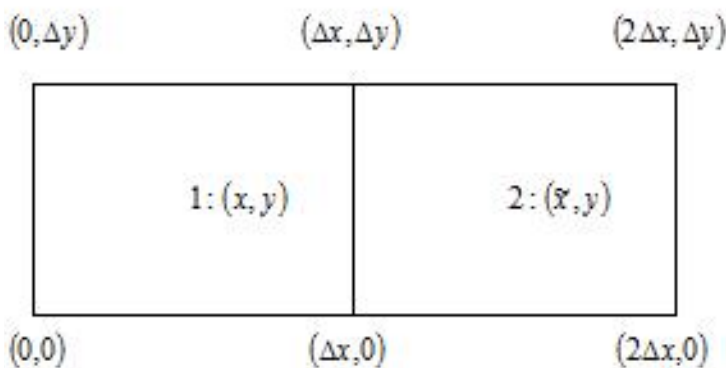

Fig. (1). Geometry of the domain of two piecewise polynomials in terms of $x$ and $y$.

where $\Delta x$ and $\Delta y$ are some constants. Likewise, the geometry in terms of $\tilde{x}$ and $y$ may be depicted as:

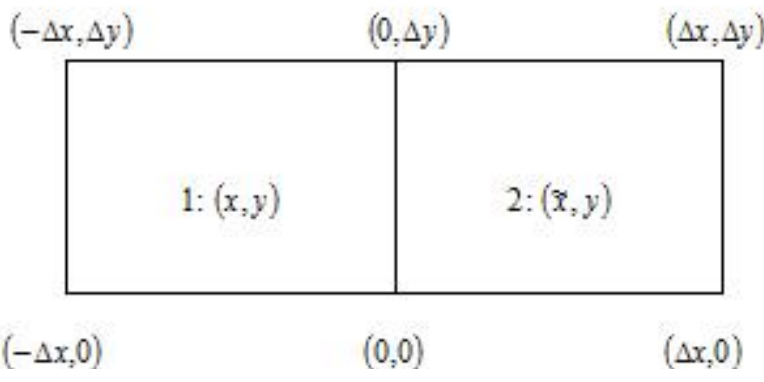

Fig. (2). Geometry of the domain of two piecewise polynomials in terms of $\tilde{x}$ and $y$.

where $\Delta x$ and $\Delta y$ are the same constants as used in Fig. (1).

Now, we may define on both coordinate systems a polynomial of order $d$ :

$$
P_{d}(x, y)=\sum_{0 \leq p+q \leq d} c_{p q} x^{p} y^{q}
$$

We start with the most simple case, that is, we set $d=1$. The polynomial equations for both coordinate systems then become: 
$z_{1}(x, y)=c_{11}+c_{12} x+c_{13} y, 0 \leq x \leq \Delta x, 0 \leq y \leq \Delta y$

$z_{2}(\tilde{x}, y)=c_{21}+c_{22} \tilde{x}+c_{23} y, 0 \leq \tilde{x} \leq \Delta x, 0 \leq y \leq \Delta y$

If we look at Fig. (2), we see that

$\tilde{x}=x-\Delta x$

Combining (2) and (3) we get:

$z_{1}(x, y)=c_{11}+c_{12} x+c_{13} y, 0 \leq x \leq \Delta x, \quad 0 \leq y \leq \Delta y$

$z_{2}(x, y)=c_{21}+c_{22}(x-\Delta x)+c_{23} y, \quad \Delta x \leq x \leq 2 \Delta x, \quad 0 \leq y \leq \Delta y$

Let

$\mathbf{z}=\left(z_{1}(x, y) \quad z_{2}(x, y)\right)^{T}$

be the outcome vector. Then (4) may be rewritten as the matrix-vector product of the polynomial base

$B=\left(\begin{array}{cccccc}1 & x & y & 0 & 0 & 0 \\ 0 & 0 & 0 & 1 & x-\Delta x & y\end{array}\right)$

and the coefficient vector

$\mathbf{c}=\left(\begin{array}{llllll}c_{11} & c_{12} & c_{13} & c_{21} & c_{22} & c_{23}\end{array}\right)^{T}$

that is,

$\mathbf{z}=B \mathbf{c}$

Note that the $(x, y)$-values that fall in the first quadrant of Fig. (1) are assigned to the first row of the polynomial base $B$, while $(x, y)$-values in the second quadrant are assigned to the second row.

\section{ENFORCING ZEROTH ORDER CONTINUITY}

In order for the two polynomials (4) to connect at the boundary, that is, in order to have $C^{0}$ continuity, we must have that

$z_{1}(\Delta x, y)=z_{2}(\Delta x, y)$

for any $y$. Substituting (4) in (7), we find

$c_{11}+c_{12} \Delta x+c_{13} y=c_{21}+c_{23} y$

or, equivalently,

$c_{11}+c_{12} \Delta x+c_{13} y-c_{21}-c_{23} y=0$

We have that (8) is a constraint on the coefficients. The coefficients c must all lie in the null-space of the smoothness "matrix" $H$, where

$H=\left(\begin{array}{llllll}1 & \Delta x & y & -1 & 0 & -1\end{array}\right)$

The null-space [4], of $H$ is

$$
H_{0}=\left(\begin{array}{ccccc}
y & 0 & 1 & -y & -\Delta x \\
0 & 0 & 0 & 0 & 1 \\
0 & 0 & 0 & 1 & 0 \\
0 & 0 & 1 & 0 & 0 \\
0 & 1 & 0 & 0 & 0 \\
1 & 0 & 0 & 0 & 0
\end{array}\right)
$$

and it may be checked that
$H H_{0}=\mathbf{0}$

where $\mathbf{0}$ is the $1 \times 5$ zero vector. It follows that the matrix product of $H$ with any linear combination of the columns in $H_{0}$ must give a zero value, that is,

$H H_{0} \mathbf{c}_{0}=0$

where $\mathbf{c}_{0}$ is an arbitrary $5 \times 1$ vector. Stated differently, any linear combination of the columns of $H_{0}$ gives us an $6 \times 1$ vector that satisfies the constraint (7) or, equivalently, constraint (8).

Now, if we take the matrix product of our original polynomial base, $B$, and the null-space of our smoothness matrix, $H_{0}$, we get the null-base $B_{0}$ :

$B_{0}=\mathrm{BH}_{0}$

$=\left(\begin{array}{ccccc}y & 0 & 1 & 0 & x-\Delta x \\ y & x-\Delta x & 1 & 0 & 0\end{array}\right)$

If we drop the zero column in (11) and rearrange the columns somewhat, we get the C-spline base, $B_{C}$ :

$B_{C}=\left(\begin{array}{cccc}1 & y & x-\Delta x & 0 \\ 1 & y & 0 & x-\Delta x\end{array}\right)$

Let

$\mathbf{b}=\left(\begin{array}{llll}b_{1} & b_{2} & b_{3} & b_{4}\end{array}\right)^{T}$

be an arbitrary coefficient vector. Then

$\mathbf{z}=B_{C} \mathbf{b}$

corresponds with the polynomial equations

$z_{1}(x, y)=b_{1}+b_{2} y+b_{3}(x-\Delta x), \quad 0 \leq x \leq \Delta x, \quad 0 \leq y \leq \Delta y$

$z_{2}(x, y)=b_{1}+b_{2} y+b_{4}(x-\Delta x), \Delta x \leq x \leq 2 \Delta x, \quad 0 \leq y \leq \Delta y$

Now, if we substitute $x=\Delta x$ in (13) we have that for any choice of $\mathbf{b}$ constraint (7) is satisfied:

$z_{1}(\Delta x, y)=z_{2}(\Delta x, y)=b_{1}+b_{2} y$

It follows that $B_{C},(12)$, is the base that enforces zeroth order continuity.

We summarize, $C^{0}$ continuity between two piecewise polynomials results in a smoothness matrix $H$, (9). The coefficients $\mathbf{c},(6)$, defined on the original polynomial base $B,(5)$, are constrained to lie within the null-space of this smoothness matrix. Stated differently, the coefficients c are constrained to be a linear combination of the columns of $H_{0}$, (10), which span the null space of $H$. By directly multiplying the null-space matrix $H_{0}$ with the the original polynomial base $B$ we get the null-base $B_{0}$ [4], which contains redundant columns consisting of zero vectors. Dropping these zero vectors we obtain the C-spline base $B_{C}$, 
(12), which has the $C^{0}$ constraint (7) build into its structure, as may be checked, (14).

\section{ENFORCING FIRST ORDER CONTINUITY}

In order for the partial derivatives of the two polynomials (4) to connect at the boundary, that is, in order to have $C^{1}$ continuity, we must have that the partial derivatives $\partial z_{1} / \partial x$ and $\partial z_{2} / \partial x$ are $C^{0}$ at their boundaries, that is

$\left.\frac{\partial z_{1}(x, y)}{\partial x}\right|_{x=\Delta x}=\left.\frac{\partial z_{2}(x, y)}{\partial x}\right|_{x=\Delta x}$

Substituting (4) in (15), we find

$c_{12}=c_{22}$

or, equivalently,

$c_{12}-c_{22}=0$

Adding constraint (16) to (9), the new smoothness matrix $H$ and the corresponding null space $H_{0}$ become, respectively,

$H=\left(\begin{array}{cccccc}1 & \Delta x & y & -1 & 0 & -1 \\ 0 & 1 & 0 & 0 & -1 & 0\end{array}\right)$

and [4],

$$
H_{0}=\left(\begin{array}{cccc}
y & -\Delta x & 1 & -y \\
0 & 1 & 0 & 0 \\
0 & 0 & 0 & 1 \\
0 & 0 & 1 & 0 \\
0 & 1 & 0 & 0 \\
1 & 0 & 0 & 0
\end{array}\right)
$$

Multiplying (17) with the original polynomial base (5) we get

$B_{0}=B H_{0}$

$=\left(\begin{array}{llll}y & x-\Delta x & 1 & 0 \\ y & x-\Delta x & 1 & 0\end{array}\right)$

Dropping the redundant zero column and rearranging the columns somewhat, we get the $\mathrm{C}$-spline base:

$B_{C}=\left(\begin{array}{ccc}1 & y & x-\Delta x \\ 1 & y & x-\Delta x\end{array}\right)$

which, since $\Delta x$ is a constant, is equivalent to the base

$B_{C}=\left(\begin{array}{ccc}1 & y & x \\ 1 & y & x\end{array}\right)$

From C-spline-base (19) it can be seen that the first order piecewise polynomials which have first order partial derivatives everywhere collapse to a global polynomial of order $d=1$ and $C^{1}$, which is just the base of a linear regression model having an intercept and predictors $x$ and $y$.

Here the given framework for deriving C-spline bases may be generalized to $d$ th order piecewise polynomials with $r$ th order continuity, $0 \leq r \leq d$, on arbitrary geometries. If one does this then it is found that the $\mathrm{C}$-spline base, $B_{C}$, has a relatively simple structure. This simple structure allows us to directly construct $B_{C}$ without first having to compute the null matrix $H_{0}$ and then taking its matrix product with the original base $B$. This makes $\mathrm{C}$-spline modeling, as given in the next section, computationally efficient. It will be seen that the computational burden of constructing a $\mathrm{C}$-spline is equivalent to that of performing an ordinary regression analysis.

\section{AN ALGORITHM TO CONSTRUCT C-SPLINES}

We give here the algorithm for the construction of Csplines for bivariate geometries, partitioned into $I \times J$ adjacent Cartesian domains on which $d$ th order piecewise polynomials with $r$ th order continuity everywhere are defined.

\subsection{The Geometry}

First we define a partitioning of the Cartesian $(x, y)$ plane. Then we number the resulting partitionings. In the region of interest the $x$ values take on values from $a_{x}$ to $b_{x}$ and the $y$ values take on values from $a_{y}$ to $b_{y}$. If we partition the $x$-axis in $I$ adjacent axes with equal lengths $\Delta x$ and the $y$-axis in $J$ adjacent axes with equal lengths $\Delta y$. Then this results in $K=I J$ partitionings.

Now, we may number each partitioning in the following manner. For $i=1$ we number the partitionings of the $y$-axis from $k=1, \ldots, J$, for $i=2$ we number the partitionings of the $y$-axis from $k=J+1, \ldots, 2 J$, etc... We then have that the $(i, j)$ th partitioning is numbered as

$k=(i-1) J+j, \quad 1 \leq i \leq I, \quad 1 \leq j \leq J$

where $k=1, \ldots, K$ and $K=I J$.

In the next paragraph we will construct our C-spline base. The geometry, as given in (20), is non-trivial in that the Cartesian coordinate system having coordinates $(i, j)$ corresponds with the $k$ th row of this C-spline base.

\subsection{Constructing the C-Spline Base}

First we construct the building blocks of our base. Let

$u_{i}=\left\{\begin{array}{cc}\left(x-a_{x}\right)-i \Delta x, & i=1, \ldots, I-1 \\ \left(x-a_{x}\right)-(i-1) \Delta x, & i=I\end{array}\right.$

Then the $x$-columns of the building blocks are: 
$u_{k i}=\left\{\begin{array}{cc}u_{i}, & k=1, \ldots, i J, \quad\{i=1, \ldots, I \\ 0, & \text { else }\end{array}\right.$

where $k=1, \ldots, K$ and $K=I J$. Likewise, let

$v_{j}=\left\{\begin{array}{cc}\left(y-a_{y}\right)-j \Delta y, & j=1, \ldots, J-1 \\ \left(y-a_{y}\right)-(j-1) \Delta y, & j=J\end{array}\right.$

Then the $y$-columns of the building blocks are:

$v_{k j}=\left\{\begin{array}{cc}v_{j}, & k=1, \ldots, j+(i-1) \times J, \\ 0, & \text { else }\end{array} \quad\left\{\begin{array}{l}j=1, \ldots, J \\ i=1, \ldots, I\end{array}\right.\right.$

where $k=1, \ldots, K$ and $K=I J$.

Using the building blocks (22) and (24), we may now construct the C-spline base $B_{C}$. Our polynomial is of order $d$, that is, let $p$ and $q$ be the powers of $x$ and $y$, respectively, then $0 \leq p+q \leq d$. Let

$U_{p}=\left\{\begin{array}{cc}u_{k l}^{p}, & p \leq r \\ \left\{u_{k 1}^{p}, \ldots, u_{k l}^{p}\right\}, & p>r\end{array}\right.$

$V_{q}=\left\{\begin{array}{cc}v_{k J}^{q}, & q \leq r \\ \left\{v_{k 1}^{q}, \ldots, v_{k J}^{q}\right\}, & q>r\end{array}\right.$

Then we take the outer product of $U_{p}$ and $V_{q}$ to get $B_{p, q}$, the C-spline equivalent of the polynomial term $x^{p} y^{q}$ :

$B_{p, q}=U_{p} \otimes V_{q}=\left\{\begin{array}{cc}u_{k 1}^{p} v_{k J}^{q}, & p \leq r, q \leq r \\ \left\{u_{k l}^{p} v_{k 1}^{q}, u_{k l}^{p} v_{k 2}^{q}, \ldots, u_{k l}^{p} v_{k J}^{q}\right\}, & p \leq r, q>r \\ \left\{u_{k 1}^{p} v_{k J}^{q}, u_{k 2}^{p} v_{k J}^{q}, \ldots, u_{k l}^{p} v_{k J}^{q}\right\}, & p>r, q \leq r \\ \left\{u_{k 1}^{p} v_{k 1}^{q}, u_{k 1}^{p} v_{k 2}^{q}, \ldots, u_{k l}^{p} v_{k J}^{q}\right\}, & p>r, q>r\end{array}\right.$

Just as the collection of terms $\left\{x^{p} y^{q}\right\}_{0 \leq p+q \leq d}$ span the polynomial $P_{d},(1)$, So the collection of column vectors

$B_{C}(x, y)=\left\{B_{p, q}\right\}_{0 \leq p+q \leq d}$

span the piecewise polynomials that make up the C-spline.

Note that for the geometry $I=2, J=1$, polynomial order $d=1$ and continuity order $r=0$, the C-spline base (28) will differ from (12) by one column permutation. Both bases may be considered equivalent though in that they both enforce constraint (7).

\subsection{Assigning Data Points to the C-Spline Base}

We have $N$ observed data points in the Cartesian $(x, y)$ plane that are related to some observed point on the $z$-axis through the unknown function $f$, that is

$$
f\left(x_{n}, y_{n}\right)=z_{n}, \quad n=1, \ldots, N .
$$

by using base (28), we approximate the unknown function $f$ with a collection of piecewise polynomials of degree $d$ that are $C^{r}$ continuous everywhere. To do this we first have to assign each data point $\left(x_{n}, y_{n}\right)$ to its corresponding partitioning. The $x$-and $y$-axes of each partitioning have, see paragraph 5.1, lengths of $\Delta x=\frac{b_{x}-a_{x}}{I}, \quad \Delta y=\frac{b_{y}-a_{y}}{J}$ we then have that for the data point $\left(x_{n}, y_{n}\right)$ which lies in the partitioning having coordinates $(i, j)$ :

$a_{x}+(i-1) \Delta x \leq x_{n} \leq a_{x}+i \Delta x$,

$a_{y}+(j-1) \Delta y \leq y_{n} \leq a_{y}+j \Delta y$ or, equivalently,

$(i-1) \leq \frac{x_{n}-a_{x}}{\Delta x} \leq i, \quad(j-1) \leq \frac{y_{n}-a_{y}}{\Delta y} \leq j$ it follows that the coordinates of the partitioning in which the data point $\left(x_{n}, y_{n}\right)$ lies may be found as

$i=\operatorname{ceil}\left(\frac{x_{n}-a_{x}}{\Delta x}\right), \quad j=\operatorname{ceil}\left(\frac{y_{n}-a_{y}}{\Delta y}\right)$

where $\operatorname{ceil}(x)$ is the function that gives the smallest integer that is greater than or equal to $x$. Substituting these coordinates in (20), we may assign the data point $\left(x_{n}, y_{n}\right)$ to its corresponding piecewise polynomial, or, equivalently, to its corresponding row $k$ in the base (28).

\section{Example}

Say, we use the C-spline base as given in (12)

$B_{C}(x, y)=\left(\begin{array}{cccc}1 & y & x-\Delta x & 0 \\ 1 & y & 0 & x-\Delta x\end{array}\right)$

where the first and second row of $B_{C}$ correspond, respectively, with the first and second partitioning of Fig. (1). Now, say we have a small dataset of $N=5$ observations $\left(x_{n}, y_{n}\right)$ having values of

$\left(x_{1}, y_{1}\right)=(1.1 \Delta x, 0.3 \Delta y)$

$\left(x_{2}, y_{2}\right)=(1.2 \Delta x, 0.7 \Delta y)$

$\left(x_{3}, y_{3}\right)=(0.1 \Delta x, 0.3 \Delta y)$

$\left(x_{4}, y_{4}\right)=(0.5 \Delta x, 0.1 \Delta y)$

$\left(x_{5}, y_{5}\right)=(1.7 \Delta x, 0.8 \Delta y)$

where $\Delta x$ and $\Delta y$ are some constants. Then, using (20) and (30), the points $\left(x_{3}, y_{3}\right)$ and $\left(x_{4}, y_{4}\right)$ are assigned to the first 
partitioning, or, equivalently, to the first row of $B_{C}$. Likewise, $\left(x_{1}, y_{1}\right),\left(x_{2}, y_{2}\right)$ and $\left(x_{5}, y_{5}\right)$ are assigned to the second partitioning, or, equivalently, to the second row of $B_{C}$ :

$$
\tilde{B}_{C}=\left(\begin{array}{c}
B_{C}^{(2)}\left(x_{1}, y_{1}\right) \\
B_{C}^{(2)}\left(x_{2}, y_{2}\right) \\
B_{C}^{(1)}\left(x_{3}, y_{3}\right) \\
B_{C}^{(1)}\left(x_{4}, y_{4}\right) \\
B_{C}^{(2)}\left(x_{5}, y_{5}\right)
\end{array}\right)=\left(\begin{array}{cccc}
1 & 0.3 \Delta y & 0 & 0.1 \Delta x \\
1 & 0.7 \Delta y & 0 & 0.2 \Delta x \\
1 & 0.3 \Delta y & -0.9 \Delta x & 0 \\
1 & 0.1 \Delta y & -0.5 \Delta x & 0 \\
1 & 0.8 \Delta y & 0 & 0.7 \Delta x
\end{array}\right)
$$

Note that use a tilde to signify a base $B_{C}$ to which data points have been assigned.

\subsection{Constructing a C-Spline}

Let $m$ be the number of columns of the C-spline base $B_{C},(28)$. Then, after we have assigned all $N$ data points to the base $B_{C}$, we get the $N \times m$ matrix $\tilde{B}_{C}$, see (31). The unknown $b$ coefficients of the C-spline, see (13), are found through least-squares estimation [5]:

$b=\left(\tilde{B}_{C}^{T} \tilde{B}_{C}\right)^{-1} \tilde{B}_{C}^{T} z$

where $z=\left(\begin{array}{lll}z_{1} & \cdots & z_{N}\end{array}\right)$ is the vector with output values, (29).

Now, say we wish to get the C-spline estimate $\hat{z}_{N+1}$ of the data point $\left(x_{N+1}, y_{N+1}\right)$. Then, using (20) and (30), we first determine the row $k$ of the base $B_{C}$, (28), that corresponds with this data point and then plug in its value. This results in the $1 \times m$ row-vector

$\tilde{B}_{C}^{(N+1)}=B_{C}^{(k)}\left(x_{N+1}, y_{N+1}\right)$

The estimate $\hat{z}_{N+1}$ is then found by simply taking the inner product of (33) and (32):
$z=\tilde{B}_{C}^{(N+1)} \cdot b$

We see that constructing a $\mathrm{C}$-spline is equivalent to performing a regression analysis.

\section{DISCUSSION}

We have introduced here Cartesian splines, or C-splines, for short. C-splines are piecewise polynomials which are defined on adjacent Cartesian coordinate systems and are $C^{r}$ continuous throughout. We have given here an algorithm that allows one to construct C-spline bases without first having to find the null-space of the corresponding smoothness matrix $H$. This makes the construction of a given $\mathrm{C}$-spline base computationally trivial since no null-space of $H$ has to be evaluated. This means that for C-splines the computational burden lies solely, just as in any ordinary regression analysis, in the evaluation of the inverse of $\tilde{B}_{C}^{T} \tilde{B}_{C}$, where $\tilde{B}_{C}$ is the matrix with the independent variables. Note that the algorithm, equations (20) through (28), may be generalized relatively easy to construct Csplines for multivariate domains.

\section{ACKNOWLEDGMENTS}

This research was partly funded by the Delft Cluster project (no. CT04.33.11), which is kindly acknowledged by the authors.

\section{REFERENCES}

[1] de Boor C. B-form basics. Geometric modeling: algorithms and new trends, In: Farin G, Ed. SIAM Publication 1987; pp. 131-48.

[2] Lai MJ, Schumaker LL. Scattered data interpolation using piecewise polynomials of degree six. SIAM Numer Anal 1997; 34: 905-21.

[3] Awanou G, Lai MJ, Wenston P. Multivariate splines for scattered data fitting and numerical solutions of partial differential equations. Wavelets and Splines. In: Chen G, Lai MJ, Eds. Athens 2005; pp. 24-75.

[4] Lay DC. Linear algebra and its applications. Reading, Massachusetts: Addison Wesley 2005.

[5] Zellner A. An introduction to Bayesian inference in econometrics. New York: J. Wiley \& Sons 1971. 